\title{
Atypical Kawasaki Disease in an Adolescent with Multivisceral Involvement
}

\author{
Zohair El Haddar (1), 1,2 Aziza El Ouali, ${ }^{1,2}$ Ayad Ghanam, ${ }^{1,2}$ Maria Rkain,,2 \\ Noufissa Benajiba, ${ }^{1,2}$ and Abdeladim Babakhouya ${ }^{1,2}$ \\ ${ }^{1}$ Department of Pediatrics, Mohammed VI University Hospital, Oujda, Morocco \\ ${ }^{2}$ Faculty of Medicine and Pharmacy, Mohammed Ist University, Oujda, Morocco
}

Correspondence should be addressed to Zohair El Haddar; zohair.el-haddar@ump.ac.ma

Received 9 June 2021; Revised 8 July 2021; Accepted 23 July 2021; Published 28 July 2021

Academic Editor: Ozgur Kasapcopur

Copyright ( 2021 Zohair El Haddar et al. This is an open access article distributed under the Creative Commons Attribution License, which permits unrestricted use, distribution, and reproduction in any medium, provided the original work is properly cited.

\begin{abstract}
Kawasaki disease (KD) is a vasculitis mostly seen in children aged less than 5 years. It can involve different organs and tissues. Its diagnosis is based on the clinical criteria of the American Heart Association (AHA). We report a case of a Moroccan adolescent with an atypical presentation of KD initially treated as typhoid fever. Gastrointestinal, renal, and pulmonary signs were the main clinical findings that made the diagnosis of KD challenging and delayed. The consequence was a severe cardiac damage with myocarditis and coronary artery dilation. $\mathrm{KD}$ is uncommon in adolescents, and it is important to recognize the atypical forms and the different presentations of KD in order to prevent the delay of diagnosis and treatment, and hence the cardiac complications.
\end{abstract}

\section{Introduction}

Kawasaki disease $(\mathrm{KD})$ is a vasculitis of an unknown etiology first described in 1967 [1]. It usually affects children younger than 5 years. Small and medium arteries are the most concerned by $\mathrm{KD}$ which makes it the first cause of pediatric acquired heart disease [2]. Its diagnosis is based on the clinical findings of the American Heart Association (AHA) [2]. The KD can affect also adolescents and even adults; the clinical profile is different with a higher frequency of atypical presentations [3]. We report a case of a Moroccan adolescent presenting an atypical form of $\mathrm{KD}$ with gastrointestinal, renal, and pulmonary involvement.

\section{Case Summary}

An 11-year-old boy was referred in January 2019 to our Department of Pediatrics because of a prolonged fever lasting 10 days associated with gastrointestinal signs resistant to antibiotics. There were no known allergies and personal or family history of prolonged fever or any systemic disease. The patient was febrile with deterioration in his general condition. Notably, acute abdominal pain associated with vomiting and diarrhea was reported one week before his admission. Therefore, the diagnosis of typhoid fever was considered, and antibiotic therapy including intravenous ceftriaxone $100 \mathrm{mg} /$ $\mathrm{kg} /$ day was started without any favorable improvement. In addition, arthralgia, hives, cough, and chest pain were also described. On physical examination, the child was asthenic with tachypnea and tachycardia. Abdominal palpation revealed a periumbilical tenderness. Furthermore, a skin rash on the chest area which had disappeared 2 days after appearing was noted. Bilateral ocular redness without any suppuration was also noticed. Moreover, cracked lips, a coated tongue, and pharyngitis (Figure 1), associated to nonpitting edema of the right foot and ankles were identified. Remarkably, periungual desquamation appeared few days after his admission (see timeline in Figure 2).

Biologically, a hypochromic microcytic anemia with hemoglobin at $9 \mathrm{~g} / \mathrm{dl}$, lymphopenia at $810 / \mathrm{mm}^{3}$, a high level of C-reactive protein (CRP) at $200 \mathrm{mg} / \mathrm{L}$, a high erythrocyte sedimentation rate (ESR) at $64 \mathrm{~mm} / \mathrm{h}$, a normal platelet 


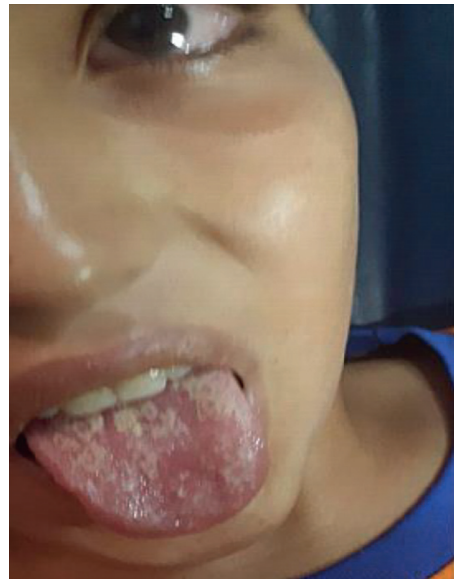

(a)

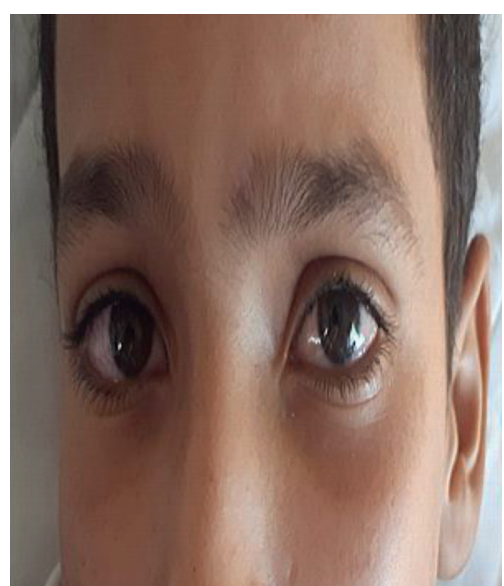

(b)

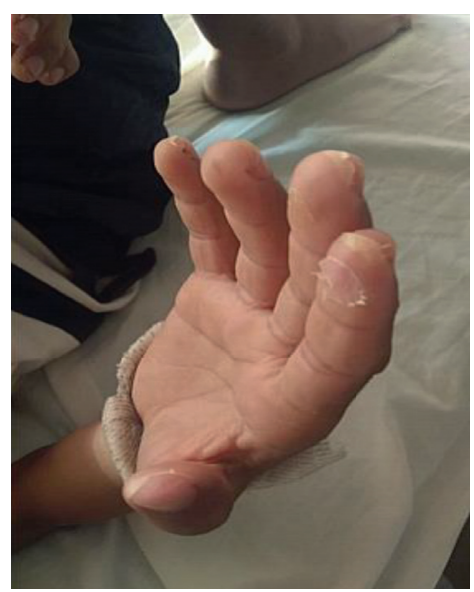

(c)

FIgUre 1: Clinical symptoms in our patient. (a) Coated tongue. (b) Bilateral ocular redness (uveitis). (c) Periungual desquamation.

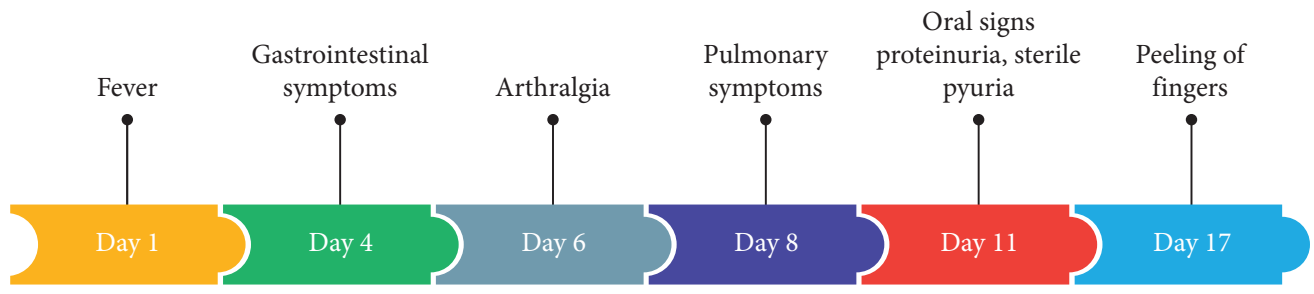

Figure 2: The timeline of the clinical case.

count at $245 \mathrm{~g} / \mathrm{l}$, and hypoalbuminemia at $26 \mathrm{~g} / \mathrm{l}$ were found. The blood culture and procalcitonin level were normal; additionally, the serologies for hepatitis A, B, C, cytomegalovirus (CMV), Epstein-Barr Virus (EBV), human immunodeficiency virus (HIV), typhoid fever, Mycoplasma pneumoniae, leptospirosis, brucellosis, rickettsia, tuberculosis, and the hemophagocytic syndrome tests were without abnormalities. Moderate proteinuria at $412 \mathrm{mg} / \mathrm{d}$ (10.3 mg/ $\mathrm{kg}$ /day) with sterile pyuria was the main renal finding. AST and ALT were <30UI/L, and complement 3 (C3), complement 4 (C4), and antinuclear antibodies were also within the normal range. However, the immunoglobulin E (IgE) level was abnormally so high at $1186 \mathrm{UI} / \mathrm{ml}$. The radiological investigations were performed with a cerebral, thoracic, and abdominopelvic computed tomography (CTAP CT) scan; the results showed inflammation of the small bowel, air-fluid levels, reactional mesenteric lymphadenopathy, and a rightsided pleurisy with bilateral pulmonary consolidation (Figure 3).

His cardiac evaluation using transthoracic echocardiogram revealed a myocarditis, pericardial effusion, mitral regurgitation, $4 \mathrm{~mm}$ coronary dilatation $(Z$ score +4$)$, and a low left ventricular ejection fraction at $43 \%$ (Figure 4 ).

Sinus tachycardia and left ventricular hypertrophy at the electrocardiography were seen. Besides, cardiac troponin was high at $397.5 \mathrm{ng} / \mathrm{ml}$ with an increased brain natriuretic peptide (BNP) at $633 \mathrm{pg} / \mathrm{ml}$. A therapeutic protocol based on intravenous immunoglobulin (IVIg) at a dose of $2 \mathrm{~g} / \mathrm{kg}$ associated with acetylsalicylic acid $100 \mathrm{mg} / \mathrm{kg} /$ day during the acute phase was started 17 days after the onset of the symptoms, then after 5 days acetylsalicylic acid $5 \mathrm{mg} / \mathrm{kg} /$ day (antiaggregating dose) per os during 3 months.

During his follow-up, the proteinuria disappeared after few days, and there were no pulmonary lesions on the CT scan control. The coronary dilation has changed from $4 \mathrm{~mm}$ to $2.5 \mathrm{~mm}$. The CT scan and the echocardiography were both performed 3 months after onset of treatment.

\section{Discussion}

Children under the age of 5 years are the most commonly affected age group by $\mathrm{KD}$ [2]. It was reported that adolescents represent only $1-7 \%$ of the patients [3-6]. Importantly and in a recent study, only $5.3 \%$ of children diagnosed with $\mathrm{KD}$ were older than 10 years [3]. In our case, the patient was 11 years old. The diagnosis of KD in its typical form is based on the clinical features of the American Heart Association (AHA) which include prolonged fever and four of the following criteria: polymorphous exanthem, extremity changes, mucosal changes involving the lips and oral cavity, bilateral bulbar conjunctival injection, and unilateral cervical lymphadenopathy [2]. Otherwise, the diagnosis of the atypical forms remains challenging for clinicians and paediatricians [3]. Up to date and to the best of our knowledge, this is the first case with concomitant pulmonary, gastrointestinal, and renal involvement to be described. 


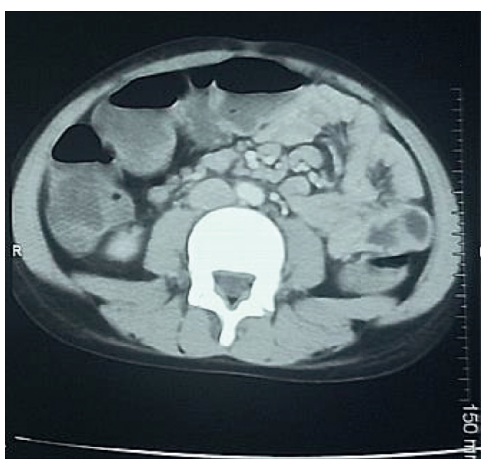

(a)

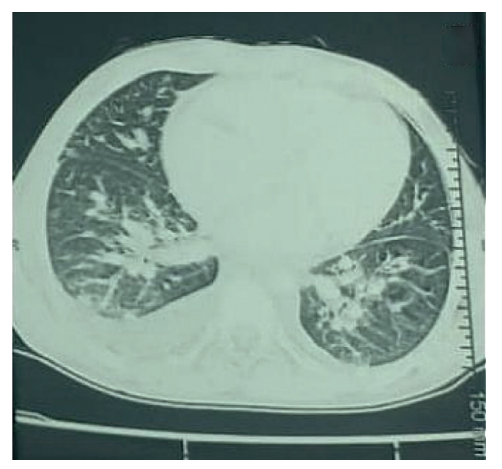

(b)

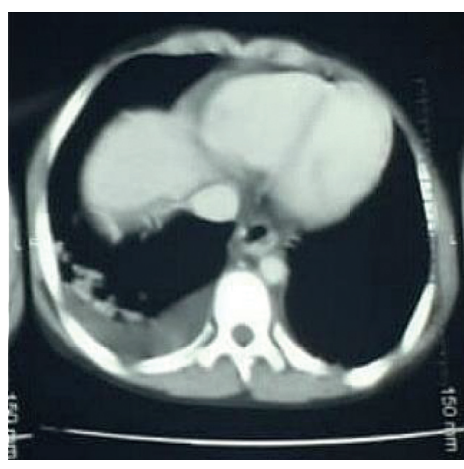

(c)

FIGURE 3: CT scan images showing (a) inflammation of the small bowel, air-fluid levels, reactional mesenteric lymphadenopathy, and (b), (c) right-sided pleurisy with bilateral pulmonary consolidation.

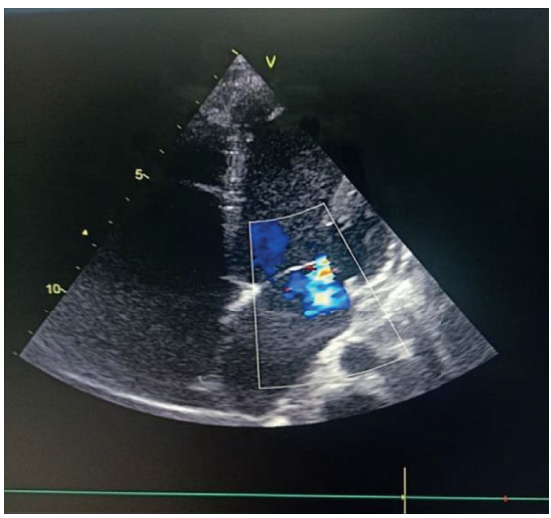

(a)

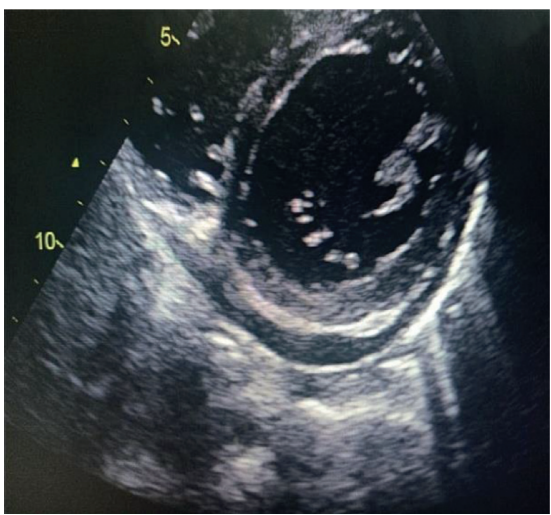

(c)

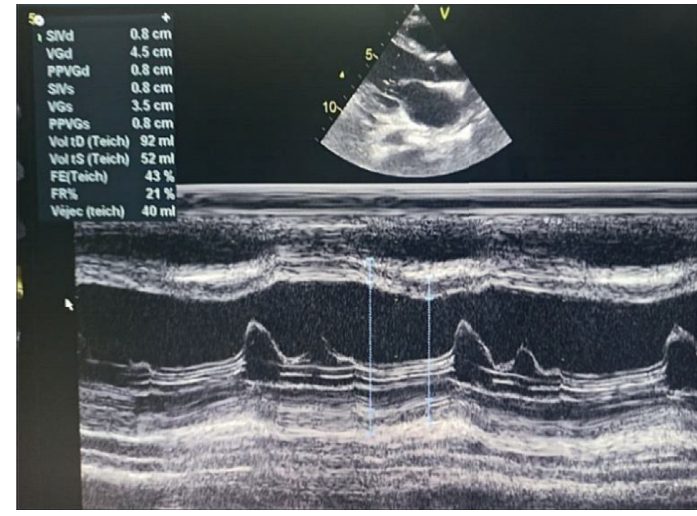

(b)

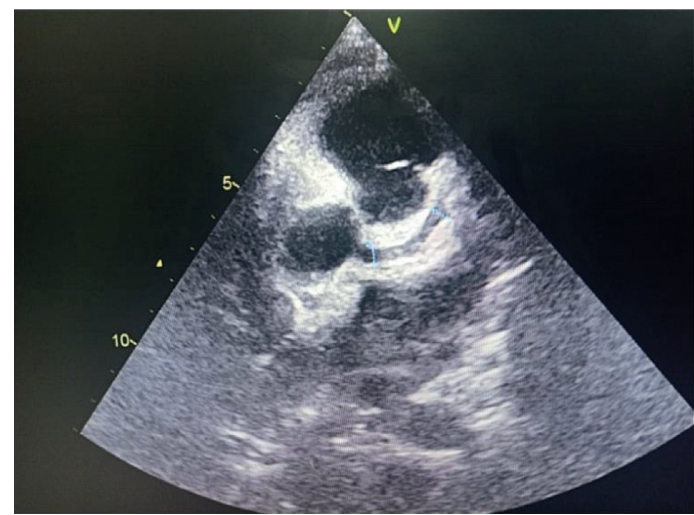

(d)

FIGURE 4: Echocardiographic images showing (a) mitral regurgitation, (b) low left ventricular ejection fraction at 43\%, (c) pericardial effusion, and (d) $4 \mathrm{~mm}$ coronary dilatation $(Z$ score +4$)$.

According to AHA recommendations, atypical $\mathrm{KD}$ is considered in children with prolonged fever and at least 2 compatible clinical criteria associated with echocardiographic findings and biological signs including late thrombocytosis, anemia, hypoalbuminemia $<30 \mathrm{~g} / \mathrm{L}$, elevated alanine aminotransferase, $\mathrm{WBC} \geq 15,000 / \mathrm{mm}^{3}$, and pyuria [2].

The echocardiographic findings supporting the diagnosis of the atypical $\mathrm{KD}$ are as follows. (i) $Z$ score of right coronary artery or left anterior descending coronary artery $\geq 2.5$

(ii) Coronary artery aneurysm

(iii) Three or more other suggestive features including $Z$ scores in the left anterior descending coronary artery or right coronary artery: 2-2.5, pericardial effusion, decreased left ventricular function, and mitral regurgitation [2] 
Different gastrointestinal manifestations in $\mathrm{KD}$ have been reported in the literature; they vary from vomiting, abdominal pain, diarrhea, abdominal distension, paralytic ileus, hepatomegaly, jaundice, and hydrops of gallbladder to less frequent findings: pancreatitis, gastrointestinal obstruction, pseudoobstruction, sigmoid colitis, and sclerosing cholangitis [7]. The prevalence of gastrointestinal clinical symptoms in $\mathrm{KD}$ is unknown [7, 8]. Miyake et al. and Fabi et al. reported, respectively, a prevalence of $23 \%$ and $35 \%$ in their studies $[8,9]$, and a high percentage was found in radiological examinations, especially of the gallbladder [10].

The gastrointestinal involvement in $\mathrm{KD}$ can lead to a delay of diagnosis and treatment, unnecessary surgical intervention, more cardiac complications, and more immunoglobulins resistance $[8,9]$. Sterile pyuria and proteinuria are the renal features in our observation and the two most common reported renal symptoms in KD [11]. The kidney inflammation may be a consequence of renal vasculitis or immune-related mechanisms [11]. Different renal disorders have been described in the literature. Chuang et al. reported an incidence of $28 \%$ of acute kidney injuries [12]. Other less common features can be found in KD: hemolytic-uremic syndrome, nephrotic syndrome, acute renal failure, renal scars, renal tubular abnormalities, immune complex-mediated nephropathy, and acute nephritic syndrome [11-16].

Pulmonary presentation of KD is exceptional. Singh et al. showed that only $1.83 \%$ of patients had a pulmonary involvement [17], while this percentage can reach $8.7 \%$ in adolescents [3]. The main symptoms are pleural effusion, pneumonia, pulmonary nodules, bronchopneumonia, and hydropneumothorax [18]. Recently, Arslanoglu Aydin et al. identified in their review of literature on pulmonary involvement in $20 \mathrm{KD}$ patients with pleural effusions [18]. Patients and especially adolescents with pulmonary involvement due to $\mathrm{KD}$ may be more likely to have atypical $\mathrm{KD}$ and coronary artery abnormalities (CAA) due to diagnosis and therapeutic delay $[3,18]$. The median interval between the onset of fever and the diagnosis of $\mathrm{KD}$ in adolescents is higher than younger children $[3,4,19]$ which was in line with our case (17 days). Compared to younger children, the adolescents had a higher incidence of atypical $\mathrm{KD}$ and also CAA $[3-5,19]$.

\section{Conclusion}

$\mathrm{KD}$ is a multisystemic vasculitis that can involve multiple organs and tissues. The diagnosis in adolescents may be delayed especially if the atypical signs are the first to appear. It must be kept in mind in its atypical form to prevent cardiac complications and especially coronary artery abnormalities.

\section{Data Availability}

The patient's data used to support the findings of this study can be retrieved from the archives of the Department of Pediatrics at the Mohammed VI University Hospital of Oujda, Morocco.

\section{Consent}

A written and signed consent was provided by the patient's family to publish the findings of this case report.

\section{Conflicts of Interest}

The authors declare that they have no conflicts of interest.

\section{References}

[1] T. Kawasaki, "Acute febrile mucocutaneous syndrome with lymphoid involvement with specific desquamation of the fingers and toes in children," Arerugi, vol. 16, pp. 178-222, 1967, in Japanese.

[2] B. W. McCrindle, A. H. Rowley, J. W. Newburger et al., "Diagnosis, treatment, and long-term management of kawasaki disease: a scientific statement for health professionals from the American heart association," Circulation, vol. 135, no. 17, pp. e927-e999, 2017.

[3] A. K. Jindal, R. K. Pilania, S. Guleria et al., "Kawasaki disease in children older than 10 years: a clinical experience from northwest India," Frontiers in Pediatrics, vol. 8, p. 24, 2020.

[4] C. Manlhiot, R. S. M. Yeung, N. A. Clarizia, N. Chahal, and B. W. McCrindle, "Kawasaki disease at the extremes of the age spectrum," Pediatrics, vol. 124, no. 3, pp. e410-e415, 2009.

[5] N. Advani, L. A. Santoso, and S. Sastroasmoro, "Profile of kawasaki disease in adolescents: is it different?" Acta Medica Indonesiana, vol. 51, no. 1, pp. 42-46, 2019.

[6] Y. Nagamori, Y. Ogihara, K. Sato, T. Ebato, K. Otani, and S. Nakamura, "Abstract 16: a clinical study of older children with Kawasaki disease," Circulation, vol. 131, no. suppl.2, p. A16, 2015.

[7] M. Fabi, E. Corinaldesi, L. Pierantoni et al., "Gastrointestinal presentation of Kawasaki disease: a red flag for severe disease?" PLoS One, vol. 13, no. 9, Article ID e0202658, 2018.

[8] C. Colomba, S. La Placa, L. Saporito et al., "Intestinal involvement in kawasaki disease," The Journal of Pediatrics, vol. 202, pp. 186-193, 2018.

[9] T. Miyake, J. Kawamori, T. Yoshida, H. Nakano, S. Kohno, and S. Ohba, "Small bowel pseudo-obstruction in Kawasaki disease," Pediatric Radiology, vol. 17, no. 5, pp. 383-386, 1987.

[10] D. Y. Yi, J. Y. Kim, E. Y. Choi, J. Y. Choi, and H. R. Yang, "Hepatobiliary risk factors for clinical outcome of Kawasaki disease in children," BMC Pediatrics, vol. 14, no. 1, p. 51, 2014.

[11] T. Watanabe, "Clinical features of acute kidney injury in patients with Kawasaki disease," World Journal of Clinical Pediatrics, vol. 7, pp. 83-88, 2018.

[12] G.-T. Chuang, I.-J. Tsai, M.-T. Lin, and L.-Y. Chang, "Acute kidney injury in patients with Kawasaki disease," Pediatric Research, vol. 80, no. 2, pp. 224-227, 2016.

[13] P. Krug, O. Boyer, E. Balzamo, D. Sidi, A. Lehnert, and P. Niaudet, "Nephrotic syndrome in Kawasaki disease: a report of three cases," Pediatric Nephrology, vol. 27, no. 9, pp. 1547-1550, 2012.

[14] M. de La Harpe, S. di Bernardo, M. Hofer, and N. Sekarski, "Thirty years of kawasaki disease: a single-center study at the university hospital of lausanne," Frontiers in Pediatrics, vol. 7, p. 11, 2019.

[15] B. Maji, S. Banerjee, and P. Pal, "Nephrotic syndrome in Kawasaki disease," Clinical Pediatrics, vol. 53, no. 9, pp. 898-899, 2014. 
[16] T. Watanabe, "Kidney and urinary tract involvement in kawasaki disease," International Journal of Pediatrics, vol. 2013, Article ID 831834, 8 pages, 2013.

[17] S. Singh, A. Gupta, A. K. Jindal et al., "Pulmonary presentation of Kawasaki disease-a diagnostic challenge," Pediatric Pulmonology, vol. 53, no. 1, pp. 103-107, 2018.

[18] E. Arslanoglu Aydin, S. Demir, O. Aydin, Y. Bilginer, and S. Ozen, "Pleural effusion as an atypical presentation of Kawasaki disease: a case report and review of the literature," Journal of Medical Case Reports, vol. 13, no. 1, p. 344, 2019.

[19] Z. Cai, R. Zuo, and Y. Liu, "Characteristics of Kawasaki disease in older children," Clinical Pediatrics, vol. 50, no. 10, pp. 952-956, 2011. 